\title{
QUARTA REVOLUÇÃO INDUSTRIAL, INTELIGÊNCIA ARTIFICIAL E A PROTEÇÃO DO HOMEM NO DIREITO BRASILEIRO
}

\author{
FOURTH INDUSTRIAL REVOLUTION, \\ ARTIFICIAL INTELLIGENCE AND THE \\ PROTECTION OF MAN IN BRAZILIAN LAW
}

DIRCEU PEREIRA SIQUEIRA ${ }^{1}$

FERNANDA CORRÊA PAVESI LARA ${ }^{2}$

\section{RESUMO}

A pesquisa tem por objetivo traçar uma aproximação entre o contexto de profunda transformação caracterizado pela Quarta Revolução Industrial e o Direito, investigando a (in)existência de regramentos legais para proteção do homem em sua individualidade, a (in)suficiência dos direitos e garantias individuais e os desafios para o estabelecimento de novos marcos legislativos. Para tanto, seguindo o método hipotético-dedutivo e como procedimentos investigativos a revisão bibliográfica em livros e periódicos sobre o tema, o trabalho apresenta-se dividido em três seções, primeiramente optou-se por delinear o contexto da Quarta Revolução Industrial e seus marcos característicos, na sequência a relação homem/máquina e os desafios do uso da inteligência artificial e na última seção são apresentados os contornos legislativos atinentes a proteção do homem. Como resultados pondera-se que o papel do Direito no contexto abordado não necessariamente será o de estabelecer novos marcos legislativos, mas, sobretudo, buscar garantias para o cumprimento dos preceitos legislativos já existentes que deverão ser reinterpretados à luz da quarta revolução industrial.

Palavras-chave: Dados pessoais. Inteligência artificial. Quarta Revolução Industrial. Direitos Fundamentais.

1 Coordenador e Professor Permanente do Programa de Doutorado e Mestrado em Direito do Centro Universitário Cesumar (UniCesumar); Pós-doutor em Direito pela Faculdade de Direito da Universidade de Coimbra (Portugal), Doutor e Mestre em Direito Constitucional pela Instituição Toledo de Ensino - ITE/Bauru, Especialista Lato Sensu em Direito Civil e Processual Civil pelo Centro Universitário de Rio Preto, Pesquisador Bolsista - Modalidade Produtividade em Pesquisa para Doutor PPD - do Instituto Cesumar de Ciência, Tecnologia e Inovação (ICETI), Professor nos cursos de graduação em direito da Universidade de Araraquara (UNIARA) e do Centro Universitário Unifafibe (UNIFAFIBE), Professor Convidado do Programa de Mestrado University Missouri State - EUA, Editor da Revista Direitos Sociais e Políticas Públicas (Qualis B1), Consultor Jurídico, Parecerista, Advogado. ORCID iD: https://orcid.org/0000-0001-9073-7759. E-mail: dpsiqueira@uol.com.br

2 Doutoranda Bolsista PROSUP/CAPES (módulo taxa) pelo Programa de Pós-Graduação em Ciências Jurídicas no Centro Universitário de Maringá - Unicesumar, tendo como linha pesquisa instrumentos de efetivação dos direitos da personalidade. Mestre em Direito Negocial pela Universidade Estadual de Londrina - UEL. Professora Coordenadora Adjunta do Curso de Direito da Pontifícia Universidade Católica do Paraná, Campus Maringá. ID Lattes: 7388198291636030 . ORCID iD: https://orcid.org/0000-0002-3121-7996. Email: fernandapavesi@hotmail.com. 
The research aims to draw an approximation between the context of profound transformation characterized by the Fourth Industrial Revolution and the Law, investigating the (in) existence of legal rules for the protection of man in his individuality, the (in) sufficiency of individual rights and guarantees and the challenges for establishing new legislative frameworks. For this purpose, following the hypothetical-deductive method and the bibliographic review in books and periodicals on the topic as investigative procedures, the research is divide into three sections. First it decide to outline the context of the Fourth Industrial Revolution and its characteristic milestones, then the man / machine relationship and the challenges of using artificial intelligence, and in the last section, the legislative outlines related to the protection of man will be presented. As results, it is considered that the role of law in the context addressed will not necessarily be to establish new legislative frameworks, but, above all, to seek guarantees for the fulfillment of existing legislative precepts which should be reinterpreted in the light of the fourth industrial revolution.

Keywords: Personal data. Artificial intelligence. Fourth Industrial Revolution. Fundamental rights.

\section{INTRODUÇÃO}

A tecnologia tem permeado o modo de vida da sociedade, está inserida no cotidiano das pessoas e mais cedo ou mais tarde promoverá a transformação da humanidade, seja nos relacionamentos interpessoais, no trabalho, nas necessidades do dia a dia, o que outrora soava como profecia a cada dia tem se concretizado e a tecnologia se apropriado das relações sociais revolucionando várias esferas da vida humana.

Diante da dinamicidade das transformações provocadas pelo impacto da tecnologia ainda não é possível estabelecer a amplitude, profundidade e a velocidade com que irão ocorrer o que demanda, no tocante a Ciência do Direito, especial atenção aos direitos e garantias fundamentais.

Assim, com o objetivo de traçar uma aproximação entre o contexto de profunda transformação caracterizado pela Quarta Revolução Industrial e o Direito, a pesquisa propõe-se a investigar a (in)existência de regramentos legais para proteção do homem em sua individualidade, a (in)suficiência dos direitos e garantias individuais e os desafios para o estabelecimento de novos marcos legislativos.

Para tanto, seguindo o método hipotético-dedutivo e adotando como procedimentos investigativos a revisão bibliográfica em livros e periódicos sobre o tema, optou-se por sistematizar o trabalho em três seções.

Primeiramente, desenvolver-se-á o contexto das profundas transformações tecnológicas denominado de Quarta Revolução Industrial adotando-se como referencial teórico o entendimento de Klaus Schab (2016; 2019), correlacionando os marcos disruptivos na existência humana com os direitos fundamentais e com o direito ao livre desenvolvimento da personalidade.

No segundo movimento, serão apresentados os contornos da inteligência artificial e a relevância da disponibilidade de dados em abundância para seus avanços destacando, por conseguinte a relação homem/máquina. Por fim, na última seção, buscar-se-á construir um panorama acerca da legislação nacional convergente a proteção do homem em sua indi- 
vidualidade mantendo o foco no contexto das profundas transformações tecnológicas ora investigado.

Assim, pretende-se descortinar caminhos para o aprofundamento científico na temática, em especial, analisando ações preditivas na seara jurídica e a importância da abertura científica do Direito para o diálogo com outras fontes.

\section{SOBRE O CONTEXTO DAS TRANSFORMAÇÓES TECNOLÓGICAS: A QUARTA REVOLUÇÃO INDUSTRIAL}

Nos últimos 250 anos, três Revoluções Industriais mudaram o mundo e a base das transformações tiveram como mote os avanços técnico-científicos. "Em cada uma delas, as tecnologias, os sistemas políticos e as instituições sociais evoluíram juntos, mudando não apenas as indústrias, mas também a forma como as pessoas se viam em suas relações umas às outras e ao mundo natural" (SCHWAB, 2019).

Para aproximação do contexto da quarta revolução industrial, buscar-se-á partir da investigação do termo exposto por Klaus Schwab (2016, p. 16), que aponta para as transformações sociais promovidas pela tecnologia como profundas, disruptivas e capazes de promover mudança de paradigma do modo de viver do homem, essa revolução é denominada pelo autor de "Quarta Revolução Industrial", marcada pela "fusão dessas tecnologias e a interação entre os domínios físicos, digitais e biológicos".

Nessa revolução, as tecnologias emergentes e as inovações generalizadas são difundidas muito mais rápida e amplamente do que nas anteriores, as quais continuam a desdobrar-se em algumas partes do mundo. A segunda revolução industrial precisa ainda ser plenamente vivida por $17 \%$ da população mundial, pois quase 1,3 bilhão de pessoas ainda não têm acesso à eletricidade. Isso também é válido para a terceira revolução industrial, já que mais da metade da população mundial, 4 bilhões de pessoas, vive em países em desenvolvimento sem acesso à internet. 0 tear mecanizado (a marca da primeira revolução industrial) levou quase 120 anos para se espalhar fora da Europa. Em contraste, a internet espalhou-se pelo globo em menos de uma década (SCHWAB, 2016, p. 17).

Os impactos da quarta revolução industrial são narrados pelo autor como sendo da mais variada ordem, na economia, nos negócios, nos governos, países, regiões, cidades, na sociedade como todo e no indivíduo, ponto de inflexão da pesquisa. Especialmente nos negócios a denominada indústria 4.0 insere-se nos processos de transformação da quarta revolução industrial e alinha-se a esse contexto, dado os avanços nos métodos de industrialização.

They need to be dealt within order to fully realise the potential of this fourth industrial revolution. Industry 4.0 will continue to embrace cuttingedge technology and techniques, and will open up new applications that will impact industrial sectors and tomorrow's complex industrial ecosystems. Advanced ICT can and will contribute to the success of Industry 4.0 (XU; XU; LI, 2018).

No âmbito do indivíduo os impactos são múltiplos e atingem fortemente a identidade e os aspectos relacionados a ela como, o senso de privacidade, a noção de propriedade, os 
padrões de consumo, o tempo destinado ao trabalho, ao lazer, o desenvolvimento da carreira profissional, o cultivo das competências profissionais, os relacionamentos e a forma como interagimos uns com os outros, são descritos por Klaus Schwab (2016, p. 99).

As extraordinárias inovações provocadas pela quarta revolução industrial, desde as biotecnológicas até aquelas da IA, estão redefinindo o que significa ser humano. Elas estão aumentando os atuais limites da expectativa de vida, saúde, cognição e competência de maneiras que antes pertenciam somente ao mundo da ficção científica. Com o avanço dos conhecimentos e das descobertas em andamento nesses campos, é fundamental que nosso foco e nosso compromisso estejam concentrados em permanentes discussões éticas e morais. Por sermos seres humanos e animais sociais, precisamos pensar individual e coletivamente sobre como responder a temas como a extensão da vida, os bebês projetados, extração de memória e muito mais (SCHWAB, 2016, p. 100).

Predizer os desdobramentos causados pela quarta revolução industrial na individualidade que cerca a vida humana é algo que intriga pesquisadores do mundo remetendo-os, por vezes, a utopias e distopias que tornam a questão ainda mais intrigante. Fato que, as transformações tendem a modificar o espaço de vida, inicialmente marcado pela relação homem/ natureza, com os incrementos tecnológicos tendem a ser marcados pela relação homem/ máquina, não qualquer máquina, mas uma capaz de desenvolver caminhos comparados a inteligência humana ${ }^{3}$.

No contexto investigado, destaca-se o direito humano ao livre desenvolvimento da personalidade que encontra suas raízes na Declaração Universal dos Direitos Humanos previsto, em especial, nos art. XXII ${ }^{4}$ art. XXIX . Reconhecido há décadas e alinhado a construção histórica de Direitos relativos à condição humana, o direito ao livre desenvolvimento da personalidade assume espaço redimensionado, posto que o ambiente propício para se desenvolver como humano poder ser único e completo, assume contornos que a sociedade precisará reconstruir, em especial, o entendimento de segurança social, esforço nacional, econômico e cultural para promoção de espaço propício para o livre desenvolvimento da personalidade.

Acerca do direito ao livre desenvolvimento da personalidade, Lixinski (2006, s/p) pondera que seu nascedouro na Declaração de Direitos Humanos consagra o direito ao auto-desenvolvimento, analisado tanto pelo aspecto privatístico quanto do aspecto público alinhado a postura do Estado de garantidor de Direitos ${ }^{6}$.

$\mathrm{Na}$ mesma trilha do direito a dignidade humana, o direito ao livre desenvolvimento da personalidade é delineado por Moreira e Alves (2015, s/p) como sendo direito,

3 Nick Bostron (2018, p. 55), define a superinteligência como "qualquer intelecto que exceda em muito o desempenho cognitivo dos seres humanos em, virtualmente, todos os domínios de interesse".

4 Conforme art. XXII, da Declaração Universal dos Direitos Humanos: "Todo ser humano, como membro da sociedade, tem direito à segurança social, à realização pelo esforço nacional, pela cooperação internacional e de acordo com a organização e recursos de cada Estado, dos direitos econômicos, sociais e culturais indispensáveis à sua dignidade e ao livre desenvolvimento da sua personalidade" (DUDH, 1948).

5 Conforme art. XXII, da Declaração Universal dos Direitos Humanos: "Todo ser humano tem deveres para com a comunidade, na qual o livre e pleno desenvolvimento de sua personalidade é possível" (DUDH, 1948).

6 Esclarece Lixinski (2006, s/p) que "As propostas para inserção do direito ao livre desenvolvimento da personalidade foram via de regra defendidas por delegados de países de orientação política socialista, numa tentativa de dar maior evidência e força aos direitos sociais, econômicos e culturais, à época uma nova construção, mesmo de certa forma resumindo-os em uma única expressão". 
Ligado à dignidade da pessoa humana, o livre desenvolvimento da personalidade expressa à ideia da pessoa decidir o seu próprio plano de vida. As noções de liberdade, autonomia e de autodeterminação constituem a essência da personalidade moral e o livre desenvolvimento da personalidade retrata justamente uma concepção dinâmica e evolutiva da personalidade humana que se desenvolve livremente por meio de atos, relações e negócios jurídicos. Este direito tutela e promove as escolhas existenciais de cada pessoa, visando a sua própria formação e preservando, assim, a sua individualidade e dignidade naquilo que o ser humano é, e naquilo que pode ser (MOREIRA, ALVES, 2015, s/p.)

Para Nery $(2015, s / p)$, tal direito está intimamente ligado a uma imensa gama de fenômenos humanos, conforme,

No que toca ao chamado direito ao desenvolvimento da personalidade (das Recht auf Entfaltung der Persönlichkeit), no extenso rol de fenômenos que sinalizam a existência de inter-relações que podem ser classificadas com esse epíteto, apontam-se: (a) liberdade de ação; (b) liberdade corporal de movimentação; (c) a atividade de força de trabalho; (d) a atividade industrial; (e) a atividade vocacional; (f) a atividade cultural; (g) liberdade de associação e reunião; (h) liberdade de expressão do pensamento; (i) a atitude e a atividade religiosa e ética, e (j) a instrução e o aproveitamento da formação (grifos no original) (NERY, 2015, s/p).

Partindo do pressuposto da subjetividade de cada ser humano e da liberdade de se constituir em sua inteireza e unicidade em um núcleo social e cultural, o contexto imprimido pela quarta revolução industrial é carreado de impactos positivos e negativos que estão em curso. Assim, percebe-se que os caminhos para se assegurar o direito ao livre desenvolvimento a personalidade perpassam, por conseguinte a garantia ao "direito sobre a individualidade (das Recht auf Individualität), celebrando uma peculiar forma de aceitar a individualidade do homem, seu caráter próprio e sua aproximação da imagem humana ideal" (NERY, 2015, s/p) grifos no original.

A individualidade, ao ver de Hubmann (apud Nery, 2015, s/p), tem três esferas distintas:

(a) a esfera individual - nome, honra, imagem física, imagem de vida, imagem de caráter, palavra falada e palavra escrita; (b) a esfera privada - aquilo que o indivíduo pode subtrair do conhecimento da mídia, e (c) a esfera secreta ações, expressões, sentimentos de que ninguém deve tomar conhecimento. (NERY, 2015, s/p)

A proteção dos pilares da individualidade da vida humana no contexto da quarta revolução industrial, devem ser preservados. Assim, denota-se que certas tecnologias possuem seus impactos na natureza humana relativamente já estudados e mapeados pela comunidade científica, ou melhor compreendidos pela academia como o caso da internet, no entanto outras por serem mais difíceis de precisar os impactos ainda não carregam a mesma clareza, como é o caso da Inteligência Artificial (IA). 


\section{A INTELIGÊNCIA ARTIFICIAL: A RELAÇÃO HOMEM/ MÁQUINA E A IMPORTÂNCIA DOS DADOS}

Segundo Dora Kaufman (2018a, p. 19), a inteligência artificial "propicia a simbiose entre o humano e a máquina ao acoplar sistemas inteligentes artificiais ao corpo humano", como próteses no cérebro, membros biônicos, dentre outros. Descreve a autora, que a relação homem/máquina é marcada pela "interação entre o homem e a máquina como duas "espécies" distintas conectadas (homem-aplicativos, homem-algoritmos de IA)".

O termo inteligência artificial foi apresentado e conceituado pela primeira vez em 1955, pelo pesquisador John McCarthy, como "a ciência e a engenharia de fazer máquinas inteligentes, especialmente programas de computador inteligentes" (apud KAUFMAN, 2018b, p. 10). Outra célebre definição foi cunhada por Russell e Norvig, como "o estudo e concepção de agentes inteligentes, onde um agente inteligente é um sistema que percebe seu ambiente e realiza ações que maximizam suas chances de sucesso" (apud KAUFMAN, 2018b, p. 10).

Analisando com vistas a complementar os conceitos acima Davi Geiger apontou que a primeira definição de McCarthy carecia da explicação acerca do que é "inteligência". Já na definição de Russell e Norvig, "faltam definições do que é "sucesso", do que é "perceber um ambiente", do que é "ação"'. Geiger compõe assim a sua definição "Inteligência Artificial é a ciência e a engenharia de criar máquinas que tenham funções exercidas pelo cérebro dos animais" (GEIGER apud KAUFMAN, 2018b, p. 10).

Certo que o incremento das pesquisas científicas e os avanços industriais na área da tecnologia tem expandido o uso da inteligência artificial que já se incorporou ao cotidiano das sociedades, as vantagens e facilidades promovidas pelos algoritmos são contrastadas aos riscos gerados pela ausência de mapeamento acerca dos rumos que a utilização da inteligência artificial poderá acarretar.

A relação homem-máquina é tão imbricada quando se refere à inteligência artificial, pois ao mesmo tempo o homem é gerador e consumidor dos dados. "O crescimento exponencial dos dados inviabiliza a programação tradicional, remetendo inevitavelmente às técnicas de aprendizado de máquinas". Dora Kaufman (2018a, p. 29), leciona que "os algoritmos de aprendizado são os casamenteiros: eles encontram produtores e consumidores um para o outro com o melhor dos dois mundos: a diversidade de opções e o baixo custo da grande escala, com o toque da personalização associado aos pequenos".

Por tal contexto, abordar a proteção à privacidade torna-se tema relevante e muito sensível. Davi Geiger (apud KAUFMAN, 2018b, p. 11), esclarece que "não é apenas uma questão de identificar algoritmos, pois o problema inclui o acesso a dados, e o uso e a distribuição de tal dados. Sim, a combinação e correlação de dados de fontes diferentes produzindo novas informações pessoais são feitas por algoritmos, que obtêm correlações estatísticas".

Estabelecer os limites entre os benefícios e malefícios dos serviços que carregam consigo a inteligência artificial, bem como analisar até que ponto invadem as esferas de privacidade dos indivíduos são discussões que merecem prosperar à medida que "o risco maior está na combinação e correlação de dados originados em distintas fontes, que geram novos dados privados (correlações estatísticas) livres de supostos acordos de privacidade" (KAUFMAN, 2018c, p. 49). 
A reflexão assume relevância eis que para o desenvolvimento da inteligência artificial em níveis de aprendizado profundo ${ }^{7}$, a abundância de dados relevantes é fundamental, nestes termos denota-se que não basta o desenvolvimento de algoritmos é fundamental que hajam dados disponíveis.

Fagan e Levmore (2019), provocam no sentido de que "a good question to ask at this point is what level of confidence should be required before decision-making is entirely outsourced to the machine or before a human overrules the Al", os autores sugerem a importância da integração entre os seres humanos e as máquinas. "In terms of $\mathrm{Al}$, and its ability to make predictive inferences, the problem of overfit is always present so long as an additional observation adds at least one new variable to consider".

There are two reasons to think that $\mathrm{Al}$ is less likely than humans to be fooled by reversal paradoxes and statistical errors. The first, as suggested earlier, is that Al can handle big data. Reversals are more likely to occur when there is a high level of variance regarding the key variable under scrutiny in the underlying population. In the presence of substantial variance, a random sample is more likely to miss critical features of the population. The initial inquiry generates a biased result when examining the relationship among variables [...] The second advantage of $\mathrm{Al}$ in this respect derives from its ability to find connections in data; it looks for things that humans do not know, or have not the energy, to examine (FAGAN; LEVMORE, 2019, p. 29 -30).

Todo movimento de expansão da inteligência artificial requer a sistematização de "novos arcabouços legais e regulatórios", para tanto, dois desafios precisam ser suplantados no âmbito dos regramentos de proteção da privacidade do indivíduo e do uso sustentável da inteligência artificial" , são eles, "o conhecimento limitado do tema pelos legisladores e a velocidade das transformações em curso" (KAUFMANN, 2018b, p. 13).

Recentemente, a Google quando questionada pela União Europeia acerca dos desafios relacionados à inteligência artificial, a resposta da empresa foi que não criassem novas leis e regulamentos que viessem a engessar o desenvolvimento tecnológico, asseveraram que “já existem muitos regulamentos e códigos legais que são de natureza neutra em tecnologia e, portanto, suficientemente amplos para serem aplicados à IA, mas vale a pena avaliar se existem lacunas no contexto de problemas concretos específicos", continuam que uma vez detectadas " quaisquer lacunas identificadas devem ser tratadas por meio de regras práticas baseadas em princípios, baseadas na legislação existente, para evitar a criação de obrigações legais excessivamente complexas ou conflitantes" (CHEE, 2020).

7 "O aprendizado profundo é o que se conhece como "IA estreita" - inteligência que coleta dados de um domínio específico e o aplica à otimização de um resultado específico. Apesar de impressionante, ainda está muito longe da "IA geral", a tecnologia para todos os fins que pode fazer o mesmo que um humano é capaz" (LEE, 2019).

8 Recomendações para o desenvolvimento da Inteligência artificial (WHITTAKER, 2018, p. 4-6). 


\section{A PROTEÇÁO JURÍDICA DOS INDIVÍDUOS FRENTE AOS AVANÇOS ADVINDOS DO USO DA INTELIGÊNCIA ARTIFICIAL}

Na seara da proteção do indivíduo, usuário das tecnologias envolvendo em especial a inteligência artificial, pode-se destacar a proteção geral já prevista no ordenamento jurídico nacional acerca da privacidade, em especial, o art. $5^{\circ}, \mathrm{X}$ da Constituição Federal.

In Brazil, the regulations applicable in the case of personal data can be found in the Federal Constitution of 1988 and provide guarantees to citizens in regards to the inviolability of privacy, the confidentiality of correspondence, data and telephone communications, except in cases where there is a judicial order that lifts these rights" (SOARES; CARABELLI, 2019). The important right to informational self-determination, which is a constitutional protection provided in art. $5, X$, of the Magna Carta, should be applied in social relations by virtue of its horizontal application by imposing the principle of maximum effectiveness of fundamental rights (SOARES; KAUFFMAN; SALES, 2019). Furthermore, the Law no. 12.965/2014, known as the Brazilian Civil Rights Framework for the Internet, in its art. 7 ensures users the rights to the use of the Internet in Brazil, amongst these rights, the inviolability of intimacy and privacy, the confidentiality of information flow for Internet communications, as well as the rights to the stored private communications. A new legislation was approved and entered into force in 2018 in order to provide greater protection to society in the face of such technological transformation, Law 13.709/2018, which regulates data protection (SOARES, et.al, 2020, p. 3)

No contexto da quarta revolução industrial novos problemas sociais oriundos do uso da inteligência artificial (IA) ou mau uso das tecnologias da informação e comunicação (TICs), acarretam a necessidade de reinterpretar a proteção dos direitos fundamentais a luz das novas demandas. Sob tal vertente, Mendes (2011, s/p) defende o direito fundamental a proteção de dados pessoais com vistas à tutela da personalidade do homem, consubstanciado dos artigos da Constituição Federal, quais sejam, "o direito material à proteção de dados pessoais, baseado no art. 5. ${ }^{\circ}$ X, da CF/1988, e a garantia instrumental para a proteção desse direito, consubstanciada na ação do habeas data (art. 5. ${ }^{\circ}$, LXXII, da CF/1988)".

A importância de proteção dos dados pessoais, "exige que o titular tenha efetivo controle sobre a circulação dos seus dados na sociedade, o que somente pode ser alcançado por meio da garantia dos direitos de acesso, cancelamento e retificação dos dados" (MENDES, $2011, \mathrm{~s} / \mathrm{p})$.

Barrientos-Parra e Melo (2008, p. 208) indicam que a base para legislação nacional deve estar alinhada a Diretriz 95/46/EC da União Europeia, que estabelece, "the protection of individuals with regard to the processing of personal data and on the free movement of such data" ${ }^{9}$ ", em especial, o autor indica a necessidade "de que a informática esteja a serviço do indivíduo e de que não atente nem contra a identidade humana, nem contra os direitos do homem, nem contra a vida privada, nem contra as liberdades individuais nem públicas".

9 Conforme tradução livre, "a proteção das pessoas em relação ao tratamento de dados pessoais e à livre circulação desses dados" (EUROPA, 1995). 
O Marco Civil da Internet, Lei $n^{\circ} 1.295$, de 23 de abril de 2014, logo no artigo primeiro indica a função da norma de estabelecer "princípios, garantias, direitos e deveres para o uso da internet no Brasil" (BRASIL, 2014), prevendo diretrizes para atuação dos entes públicos do Estado nesse campo pode ser considerada a primeira legislação atinente a proteção de dados no Brasil. Tanto no art. $3^{\circ}$ do Marco Civil da Internet ${ }^{10}$, quanto no art. $7^{\circ}$ da referida lei denota-se a preocupação do legislador nacional convergente a proteção de dados pessoais.

Avançando na garantia do direito fundamental à proteção dos dados pessoais, a Lei $\mathrm{n}^{\circ}$ 13.709 de 14 de agosto de 2018, denominada de Lei Geral de Proteção de Dados Pessoais (LGPD) estabelece logo no primeiro artigo seu objetivo central, qual seja, o de "proteger os direitos fundamentais de liberdade e de privacidade e o livre desenvolvimento da personalidade da pessoa natural" (BRASIL, 2018). No entanto, a lei que tinha vigência prevista para agosto de 2020 foi alterada pela Medida Provisória n 959, de 29 de abril de 2020, ampliando a vacatio legis para 03 de maio de 2021 (BRASIL, 2020).

No Brasil, especificamente sobre a inteligência artificial tramitam no Congresso Nacional o Projeto de Lei $n^{\circ} 5051$, de 2019, que "estabelece os princípios para o uso da Inteligência Artificial no Brasil" (BRASIL, 2019a) e visa estabelecer a regulamentação da IA no território nacional e o Projeto de Lei $n^{\circ} 5691$, de 2019, que propõe instituir uma Política Nacional de Inteligência Artificial, "com o objetivo de estimular a formação de um ambiente favorável ao desenvolvimento de tecnologias em Inteligência Artificial" (BRASIL, 2019b).

Por ora, os projetos em apreço estão em fase de discussão e as possíveis ameaças de lesão a bens juridicamente tutelados relativas à ausência de controle da inteligência artificial seja na violação dos direitos de privacidade, seja nas decisões automáticas discriminatórias ou sem motivação e explicação, por exemplo, deverão ser resguardadas pelo arcabouço legislativo vigente.

No entanto, é preciso avançar no caminho para desenvolvimento de uma "legislação que proteja a sociedade sem impedir a inovação; proibições genéricas baseadas em medos abstratos só aumentarão a burocracia e reduzirão a produtividade. Uma boa legislação deve incentivar o progresso e evitar as ameaças" (COZMAN, 2018, p. 39).

Cabe não olvidar que encontrar o liame entre a proteção legislativa, o progresso e o incremento da inteligência artificial exigirá esforços conjuntos das várias esferas da sociedade, caminhos que deverão ser trilhados tanto no plano nacional, como em alinhamento com o plano internacional de cooperação para o desenvolvimento sustentável da IA.

10 Conforme Art. $3^{\circ} \mathrm{A}$ disciplina do uso da internet no Brasil tem os seguintes princípios: I - garantia da liberdade de expressão, comunicação e manifestação de pensamento, nos termos da Constituição Federal; II - proteção da privacidade; III - proteção dos dados pessoais, na forma da lei; IV - preservação e garantia da neutralidade de rede; V - preservação da estabilidade, segurança e funcionalidade da rede, por meio de medidas técnicas compatíveis com os padrões internacionais e pelo estímulo ao uso de boas práticas; VI - responsabilização dos agentes de acordo com suas atividades, nos termos da lei; VII - preservação da natureza participativa da rede; VIII - liberdade dos modelos de negócios promovidos na internet, desde que não conflitem com os demais princípios estabelecidos nesta Lei. Parágrafo único. Os princípios expressos nesta Lei não excluem outros previstos no ordenamento jurídico pátrio relacionados à matéria ou nos tratados internacionais em que a República Federativa do Brasil seja parte (BRASIL, 2014). 


\section{CONSIDERAÇÓES FINAIS}

Denota-se que as transformações tecnológicas oriundas da quarta revolução industrial apontam para o protagonismo da Ciência do Direito no enfrentamento da proteção da singularidade da vida humana, das garantias para espaço social seguro para o livre desenvolvimento da personalidade. Entretanto, pondera-se que o papel do Direito no contexto abordado, não necessariamente será o de estabelecer novos marcos legislativos, mas, sobretudo, buscar garantias para o cumprimento dos preceitos legislativos já existentes, que deverão ser reinterpretados à luz da quarta revolução industrial.

As estratégias do Estado via instituição de políticas públicas convergentes à proteção dos indivíduos frente às incertezas da inteligência artificial podem funcionar com importante artifício para enfrentamento sistêmico da desigualdade e riscos potenciais de agravamento da mesma em decorrência da IA e do próprio contexto social desenhado pela quarta revolução industrial.

\section{REFERÊNCIAS}

ALVES, Fernando de Brito; RIGÃO, Livia Carla Silva. Cultura da periferia e as canções de rap: um olhar para as "vozes silenciadas" a partir da filosofia de Enrique Dussel. Revista Direitos Sociais e Políticas Públicas - Unifafibe. V. 8, N. 1, 2020.

BARRIENTOS-PARRA, Jorge; MELO, Elaine Cristina Vilela Borges. O direito à intimidade na sociedade técnica: Rumo a uma política pública em matéria de tratamento de dados pessoais. Revista de Informação Legislativa. Brasília, a. 45. n. 180, out./dez. 2008. p. 197-213.

BOSTROM, Nick. Superinteligência: caminhos, perigos, estratégias. Rio de Janeiro: DarkSide Books, 2018.

BRASIL. Lei n 12.965, de 23 de abril de 2014. Estabelece Princípios, Garantias, Direitos e Deveres para o Uso da Internet no Brasil. Brasília, DF, 23 abr. 2014. Disponível em: http://www.planalto.gov.br/ccivil_03/_ato20112014/2014/lei/l12965.htm. Acesso em: 10 de jun. 2020.

BRASIL. Lei n 13.709, de 14 de agosto de 2018. Lei Geral de Proteção de Dados Pessoais (LGPD). Brasília, DF, 14 ago. 2018. Disponível em: http://www.planalto.gov.br/ccivil_03/_ato2015-2018/2018/lei//13709.htm. Acesso em: 10 de jun. 2020.

BRASIL. Presidência da República. Medida Provisória n 959. Brasília, DF, 29 abr. 2020. Disponível em: http:// www.planalto.gov.br/ccivil_03/_Ato2019-2022/2020/Mpv/mpv959.htm\#art4. Acesso em: 10 de jun. 2020.

BRASIL. Senado Federal. Projeto de Lei n 5051, de 2019 - Estabelece princípios para o uso da Inteligência Artificial no Brasil. Senador Styvenson Valentim (PODEMOS/RN). Disponível em: https://legis.senado.leg.br/sdleg-getter/documento?dm=8009064\&ts=1582300610026\&disposition=inline Acesso em 08 jun. 2020.

BRASIL. Senado Federal. Projeto de Lei n 5691, de 2019 - Estabelece princípios para o uso da Inteligência Artificial no Brasil. Senador Styvenson Valentim (PODEMOS/RN). Disponível em: https://legis.senado.leg.br/sdleg-getter/documento?dm=8031122\&ts=1582300641960\&disposition=inline Acesso em 08 jun. 2020

CASTRO, Alexander; NASCIMENTO, Gabriel Bassaga. Liberdade de expressão frente à liberdade religiosa: direitos fundamentais em conflito e proteção de direitos da personalidade frente a discursos de ódio. Revista Direitos Sociais e Políticas Públicas - Unifafibe. V. 7, N. 3, 2019.

CHEE, Foo Yun. Google quer que UE não crie novas leis sobre IA. Reuters. Brasil, 28 maio, 2020. 
COSTA, Fabrício Veiga; PINTO, Alisson Alves. A ressocialização do detento a partir do prazo para o cumprimento da função social da empresa na sociedade contemporânea. Revista Direitos Sociais e Políticas Públicas - Unifafibe. V. 7, N. 3, 2019.

COZMAN, Fabio G. Inteligência Artificial: uma utopia, uma distopia. Teccogs: Revista Digital de Tecnologias Cognitivas, TIDD | PUC-SP, São Paulo, n. 17, p. 32-43, jan-jun. 2018. Disponível em: https://www.pucsp.br/pos/tidd/ teccogs/edicao_completa/teccogs_cognicao_informacao-edicao_17-2018-completa.pdf. Acesso em: 09 de jun. 2020.

DUDH. Declaração Universal dos Direitos Humanos. Comitê de Redação da Declaração Universal dos Direitos Humanos. 10 dez. 1948.

EUROPA. Directive 95/46/EC. European Parliament and of the Council of 24 October 1995 on the protection of individuals with regard to the processing of personal data and on the free movement of such data. Official Journal L 281 , 23/11/1995 p. 0031 - 0050. Disponível em: https://eur-lex.europa.eu/eli/dir/1995/46/oj. Acesso em: 15 dez. 2019.

FAGAN, Frank; LEVMORE, Saul. The Impact of Artificial Intelligence on Rules, Standards, and Judicial Discretion. Southern California Law Review, v. 93, n. 1, pp. 1, 2019; U of Chicago, Public Law Working Paper No. 704; University of Chicago Coase-Sandor Institute for Law \& Economics Research Paper No. 876. Available at SSRN: https://ssrn. com/abstract=3362563 or http://dx.doi.org/10.2139/ssrn.3362563

KAUFMAN, Dora. Dossiê: Deep learning: a Inteligência Artificial que domina a vida do século XXI. Teccogs: Revista Digital de Tecnologias Cognitivas, TIDD I PUC-SP, São Paulo, n. 17, p. 17-30, jan-jun. 2018a. Disponível em: https:// www.pucsp.br/pos/tidd/teccogs/edicao_completa/teccogs_cognicao_informacao-edicao_17-2018-completa. pdf. Acesso em: 08 de jun. 2020.

KAUFMAN, Dora. Entrevista com Davi Geiger. Teccogs: Revista Digital de Tecnologias Cognitivas, TIDD I PUC-SP, São Paulo, n. 17, p. 10-15, jan-jun. 2018b. Disponível em: https://www.pucsp.br/pos/tidd/teccogs/edicao_completa/teccogs_cognicao_informacao-edicao_17-2018-completa.pdf. Acesso em: 08 de jun. 2020.

KAUFMAN, Dora. O protagonismo dos algoritmos da Inteligência Artificial: observações sobre a sociedade de dados. Teccogs: Revista Digital de Tecnologias Cognitivas, TIDD I PUC-SP, São Paulo, n. 17, p. 44-58, jan-jun. 2018c. Disponível em: https://www.pucsp.br/pos/tidd/teccogs/edicao_completa/teccogs_cognicao_informacao-edicao_17-2018-completa.pdf. Acesso em: 08 de jun. 2020.

LAZCANO, Alfonso Jaime Martínez. El derecho convencional y los retos de su implementación en los estados parte. Revista Direitos Sociais e Políticas Públicas - Unifafibe. V. 7, N. 3, 2019.

LIXINSKI, Lucas. Considerações acerca da inserção dos direitos de personalidade no ordenamento privado brasileiro. Revista de Direito Privado, Thomson Reuters. v. 27, 2006. p. 201-222, Jul - Set, 2006.

LOZANO, Luis Gerardo Rodrígues. León duguit y el servicio público: ideas para el siglo XXI. Revista Direitos Sociais e Políticas Públicas - Unifafibe. V. 8, N. 1, 2020.

LUCAS, Doglas Cesar. Direitos humanos, identidade e a política de reconhecimento de Charles Taylor. Revista Direitos Sociais e Políticas Públicas - Unifafibe. V. 7, N. 3, 2019.

MAGLIACANE, Alessia. L'armee des reserves dans la mondialisation : la parabole de la femme italienne de la constitution au post-fordisme. Revista Direitos Sociais e Políticas Públicas - Unifafibe. V. 7, N. 3, 2019.

MARTíN, Ignacio Durbán Origen y fundamentos del sistema plurilegislativo civil español. Revista Direitos Sociais e Políticas Públicas - Unifafibe. V. 8, N. 1, 2020.

MENDES, Laura Schertel. O direito fundamental à proteção de dados pessoais. Revista de Direito do Consumidor, Thomson Reuters. v. 79, 2011. p. 45-81, Jul - Set., 2011. DTR\2011\2474.

MORAES, Maria Valentina de; LEAL, Mônia Clarissa Hennig. Supremo tribunal federal e diálogo institucional: há um controle jurisdicional de políticas públicas no brasil? Revista Direitos Sociais e Políticas Públicas - Unifafibe. V. 7, N. 3, 2019

MOREIRA, Rodrigo Pereira; ALVES. Direito ao esquecimento e o livre desenvolvimento da personalidade da pessoa transexual. Revista de Direito Privado, Thomson Reuters. v. 64, 2015. p. 81-102, Out - Dez., 2015.DTR\2016\136. 
NERY. Rosa Maria Barreto Borriello de Andrade. Distinção entre "personalidade" e "direito geral de personalidade" uma disciplina própria. Aula inaugural proferida no Curso de Pós-graduação em Direito da PUC-SP, em 18.08.2014, na disciplina Direito de personalidade, proteção de dados e tecnologias informativas - $2 .^{\circ}$ semestre de 2014. Doutrinas Essenciais de Direito Constitucional, Thomson Reuters. v. 8, 2015. p. 473-478, Ago., 2015. DTR $2015 \backslash 11483$.

SCHWAB, Klaus. A quarta revolução industrial. Trad. Daniel Moreira Miranda. São Paulo: Edipro, 2016.

SCHWAB, Klaus. Aplicando a quarta revolução industrial. Trad. Daniel Moreira Miranda. São Paulo: Edipro, 2019.

SOARES, Marcelo Negri; Kauffman, M. E.; Chao, K. M.; Saad, M. O. New Technologies and the Impact on Personality Rights in Brazil. Pensar-Revista de Ciências Jurídicas, 2020, 25.1.

WHITTAKER, Meredith; et al. Al Now Report 2018. IA NOW. Disponível em : https://ainowinstitute.org/AI_ Now_2018_Report.pdf Acesso em 08 de jun. de 2020.

XU, Li da; XU, Eric L.; LI, Ling. Industry 4.0: state of the art and future trends. International Journal Of Production Research, [s.I.], v. 56, n. 8, p. 2941-2962, 9 mar. 2018. Informa UK Limited. http://dx.doi.org/10.1080/00207543.2 018.1444806

Recebido/Received: 28.08.2020.

Aprovado/Approved: 26.12.2020. 